\title{
Light emission and detection by metal oxide silicon tunneling diodes
}

\author{
C. W. Liu, M. H. Lee, C. F. Lin, I. C. Lin, W. T. Liu, and H. H. Lin \\ Department of Electrical Engineering, National Taiwan University, Taipei, Taiwan
}

\begin{abstract}
Both NMOS and PMOS light-emitting diodes and photodetectors are demonstrated. For the ultrathin gate oxide, the tunneling gate of metal oxide silicon (MOS) diodes can be utilized as both emitters for light emitting devices and collectors for light detectors. An electron-hole plasma model is used to fit the emission spectra. A surface band bending is responsible for the bandgap reduction in electroluminescence (EL) from the MOS tunneling diode. The dark current of the photodetectors is limited by the thermal generation of minority carrier in the inversion layer. The high growth temperature $\left(1000^{\circ} \mathrm{C}\right)$ of the oxide can reduce the dark current to a level as low as $3 \mathrm{nA} / \mathrm{cm}^{2}$.
\end{abstract}

\section{Introduction}

It is a long sought goal to integrate ultra large scale integrated circuits(ULSI) with the electro-optics to possibly overcome the speed limitation of electrical interconnects and to reduce the complexity of the back-end process at the same time. Light emitting diodes and detectors are essential devices for this application. An avalanche diode and a $\mathrm{SiGe/Si} \mathrm{p-i-n} \mathrm{diode} \mathrm{have} \mathrm{been} \mathrm{used} \mathrm{as} \mathrm{a} \mathrm{light} \mathrm{emitter} \mathrm{to} \mathrm{serve}$ this purpose $[1,2]$. In this paper, a MOS tunneling diode biased at the accumulation region is used as a light emitting device and a MOS tunneling diode biased at the inversion region is used as a photodetector. Both are fully compatible with CMOS technology. The same device structure for emitters and detectors makes it possible to have a duplex mode of optical interconnects. On the other hand, the conventional CCD imager requires a serial readout and thus reduces readout speed. The tunneling gate current from the MOS diode biased in the inversion region can be used as a photo signal and can be read in parallel.

\section{Fabrication}

The ultra thin gate oxide used in the study is grown by rapid thermal oxidation (RTO) at the $900-1000^{\circ} \mathrm{C}$. The gas flows are $500 \mathrm{sccm}$ nitrogen and $500 \mathrm{sccm}$ oxygen at a reduced pressure. Before oxidation, the sample was cleaned by a HF dip and an in-situ hydrogen bake. After the growth of the ultra thin oxide, the sample was in-situ annealed in nitrogen for $10 \mathrm{~min}$ at $900^{\circ} \mathrm{C}$. The thickness is measured by ellipsometer, confirmed by $\mathrm{x}$-ray reflectivity and high frequency $\mathrm{C}-\mathrm{V}$ measurements. The resistivities of the 100 $\mathrm{mm}$ p-type wafer and the $100 \mathrm{~mm}$ n-type wafer used in this study are 1-5 and 1-10 ohm-cm, respectively. The MOS diodes had $\mathrm{Al}$ or indium tin oxide(ITO) gate electrodes with various circular areas defined by photolithography.

\section{Light emitting diode}

For NMOS diodes, the negative gate voltage will inject electrons from metal to silicon and attract the positive localized holes in the silicon/oxide interface (accumulation layer). Fig. 1 shows the $\mathrm{I}-\mathrm{V}$ curves of an $\mathrm{Al} / 2.3 \mathrm{~nm}$ oxide/p-Si device with oxide grown at $900^{\circ} \mathrm{C}$. Fig. 2 plots the $\mathrm{C}-\mathrm{V}$ curve of this NMOS diode. The diode area is $3 \times 10^{-4} \mathrm{~cm}^{2}$. Using the equivalent circuit inserted in Fig.2, the thickness extracted from the $\mathrm{C}-\mathrm{V}$ is about $2.6 \mathrm{~nm}$, similar to the $2.3 \mathrm{~nm}$ measured by ellipsometer. The FN or direct tunneling current of electrons at the negative bias can cause electron hole recombination in the silicon/oxide interface as shown in Fig.3. The interface roughness and spread in k-space due to localized holes in the accumulation layer can provide the extra momentum to emit the photons. Luminescence was clearly observed at the negative gate bias under continuous wave operation without cooling. Since the light was blocked by the thick $\mathrm{Al}$ gate electrode, only the emission from the diode edge was detected, as observed by IR viewer through a microscope. The photon emission spectra of a NMOS with a area of $4 \times 10^{-2} \mathrm{~cm}^{2}$ (Fig.4) have peaks at around $1.06 \mathrm{eV}$ for gate voltage from negative 2.5 to $4 \mathrm{~V}$, and the intensity increases as the voltage increases. Note that no light comes out from the diodes at positive gate bias.

Fig. 5 shows the typical I-V curves of a PMOS diode with the oxide thickness of $2.7 \mathrm{~nm}$. When the Al gate with the area of $3 \times 10^{-4} \mathrm{~cm}^{2}$ was under positive bias, the electrons tunnel from the n-type silicon to the $\mathrm{Al}$ gate and the holes also tunnel from $\mathrm{Al}$ gate to $\mathrm{n}$-type silicon. As a result, a significant tunneling current was observed in the positive gate bias. However, due to the different barrier heights between electrons $(\sim 3.1 \mathrm{eV})$ and holes $(\sim 5.6 \mathrm{eV})$, hole current may be smaller than the electron current (Fig.6). Meanwhile, the positive gate bias also attracts electrons in the silicon/oxide interface to form an accumulation layer. Similarly, the localized electrons in the accumulation layer with localized wave functions and the $\mathrm{Si}$ /oxide interface roughness may provide extra momentum during the electron-hole radiative recombination. For the PMOS diode, intensive light (Fig.7) is also emitted by PMOS tunneling diode with a peak around $1.05 \mathrm{eV}$ for gate bias from positive $3 \mathrm{~V}$ to $5 \mathrm{~V}$. The emission intensity increases with the positive gate bias. The feature of the emission spectra is the asymmetrical broadening of the

\subsection{1}


emission peak, where the high-energy part is more broad than the low-energy part. This is very similar to the line shape of electron-hole-plasma (EHP) recombination. To confirm this observation, we fit the EL spectra by the line shape of EHP recombination [3]. Fig.7 also shows the theoretical EHP spectra, indicating an excellent fit to the experimental data. The bandgap obtained from the EHP model is about $1.01 \mathrm{eV}$ to $1.03 \mathrm{eV}$ for the gate voltage from $5 \mathrm{~V}$ to $3 \mathrm{~V}$, lower than the Si bandgap of $1.12 \mathrm{eV}$ at room temperature. This is unlikely due to high junction temperature in $\mathrm{Si} /$ oxide interface, since the temperature of $1.01 \mathrm{eV}$ bandgap is about $600 \mathrm{~K}$ and the EL spectra can not be fitted well using such high temperature. This reduction of bandgap can be understood by the surface band bending in the PMOS band diagram under the accumulation condition as shown in Fig. 6. The tunneling holes from the $\mathrm{Al}$ gate electrode injected into the valence band of $\mathrm{n}-\mathrm{Si}$ will not stop at the interface, can probably reach the neutral region of $\mathrm{n}-\mathrm{Si}$, and relaxed to the valence band edge in the neutral region. The recombination of holes in the neutral region with the electrons in the accumulation region can emit photons with energy of the difference between the Si bandgap and the surface band bending. Similar results are also obtained from NMOS (Fig. 4).

\section{Photodetector}

When the gate is biased at the positive voltage greater than threshold voltage in a NMOS tunneling diode, an inversion layer of electrons is formed at the oxide/p-silicon interface. As the positive gate bias increases, more and more electrons tunnel from the "incipient" inversion layer to the $\mathrm{Al}$ electrode through the thin gate oxide. Finally, the electron current saturates for the gate biased at a large enough voltage $(0.3 \mathrm{~V})$, since the current is limited by the generation rate of the electrons in the inversion layer (Fig.8). When photons with energy larger than the bandgap illuminate on the diode, the photo-generated electrons in the p-silicon diffuse, and are swept by electric field to the $\mathrm{Al}$ electrode by tunneling through the ultra thin gate oxide. The measured detection spectrum has a cut-off wavelength at $1.18 \mu \mathrm{m}$ (Fig. 9). The absorption below the bandgap may be due to the surface band bending and/or interface states. Metal halide lamps with spectra similar to the sun are used to generate photocurrent. The photocurrent increases with the light intensity and is relatively independent of the positive gate bias (Fig. 1). The dark current can be reduced by a factor of 100 by increasing the growth temperature of rapid thermal oxide from 900 to $1000^{\circ} \mathrm{C}$ (Fig.10). The lowest value of dark current is $3 \mathrm{nA} / \mathrm{cm}^{2}$.

PMOS detector was fabricated with a similar dark current level biased at negative bias. The photo-generated holes can tunnel through ultra-thin oxide and represent the photo signal. A large saturation voltage $(-2 \mathrm{~V})$ is observed in the PMOS due to the large valence band discontinuity, as compared to NMOS. The bias-independent gate current consists of thermal-generated hole current from $\mathrm{n}-\mathrm{Si}$ to $\mathrm{Al}$ and tunneling electron current from $\mathrm{Al}$ to $\mathrm{n}-\mathrm{Si}$. The electron current may be larger than the hole current. Since hole concentration (the minority carriers in the $\mathrm{n}$ - $\mathrm{Si}$ ) in the inversion region can not reach the strong inversion, and actually decreases as the magnitude of gate bias increases (due to the increase of the tunneling probability), the oxide voltage is some sort "pinned" with deep depletion region formed in the $\mathrm{n}-\mathrm{Si}$. The photon excitation increases the hole concentration in the inversion layer at $\mathrm{n}$-Si/oxide interface, and thus increases the "pinned" oxide voltage, and finally increases the gate current. The similar effect can occur in NMOS, but is not very significant, since the thermal generated electron current from the $\mathrm{p}$-Si to the $\mathrm{Al}$ gate is larger than the tunneling hole current from the $\mathrm{Al}$ gate to the p-Si.

\section{ITO gate}

The NMOS diode using transparent electrodes (ITO) has a work function about $0.5 \mathrm{eV}$ lower than $\mathrm{Al}$ as measured by $\mathrm{C}$ V (Fig. 11). At the same negative gate bias, the ITO gate has a smaller tunneling current with similar IR spectra. The photocurrent of ITO detectors increases by a factor of 20 (Fig. 12 ), as compared to $\mathrm{Al}$ devices. The large photocurrent was observed to damage the device by slightly increasing the dark current.

\section{Conclusions}

The MOS tunneling diode can serve as both a light emitter and a light detector at a suitable bias. This configuration can be applied in a duplex mode of optical interconnects in ULSI chips as well as the image sensor. The surface band bending is responsible for the bandgap reduction in the sharp emission spectra. High growth temperature of the oxide of the photodetector can reduce the dark current to $3 \mathrm{nA} / \mathrm{cm}^{2}$.

\section{Acknowledgments}

This work is supported by National Science Council, Taiwan, R.O.C. under contract numbers: 88-2218-E-002-004, 882218-E-002-004, 88-2215-E-002-021 and 88-2112-M-002038. The initial support of UMC and TSMC is also highly appreciated.

\section{References}

[1] K. Misiakos et al., "Monolithic integration of light emitting diodes detectors, and optical fiber on a silicon wafer: a CMOS compatible optical sensor," IEDM, Tech. Dig. pp.25-28, 1998

[2] $\mathrm{Q}$. Mi et al. "Room-temperature $1.3 \mu \mathrm{m}$ electro-luminescence from strained SiGe/Si quantum wells," Appl. Phys. Lett. 60, pp.3177-3179, 1992. [3] C. W. Liu et al. " Room-temperature electroluminescence from electronhole plasmas in the metal oxide silicon tunneling diodes," will submit to Appl. Phys. Lett. 1999. 


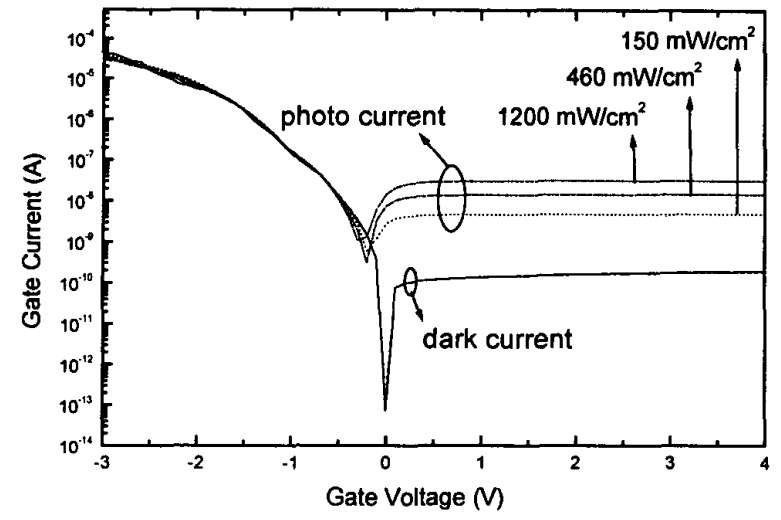

Fig.1. Photo and dark currents of a NMOS tunneling diode. Dark current does not change after light exposure. Device size is $3 \times 10^{-4} \mathrm{~cm}^{2}$. The photo current was generated by metal halide lamps with spectra similar the sun.

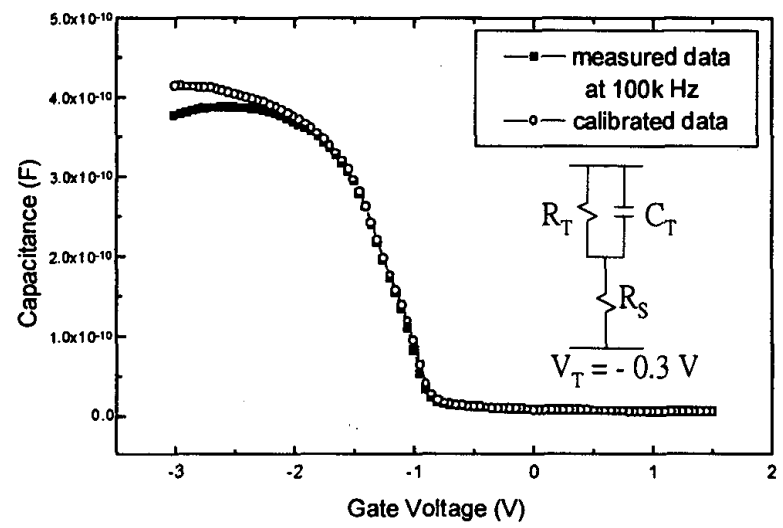

Fig. 2. A C-V plot of a NMOS tunneling diode. The thickness measured by ellipsometer is about $2.3 \mathrm{~nm}$. The thickness extracted from $\mathrm{C}-\mathrm{V}$ is $2.6 \mathrm{~nm}$. The insert is the equivalent circuit model for extraction.

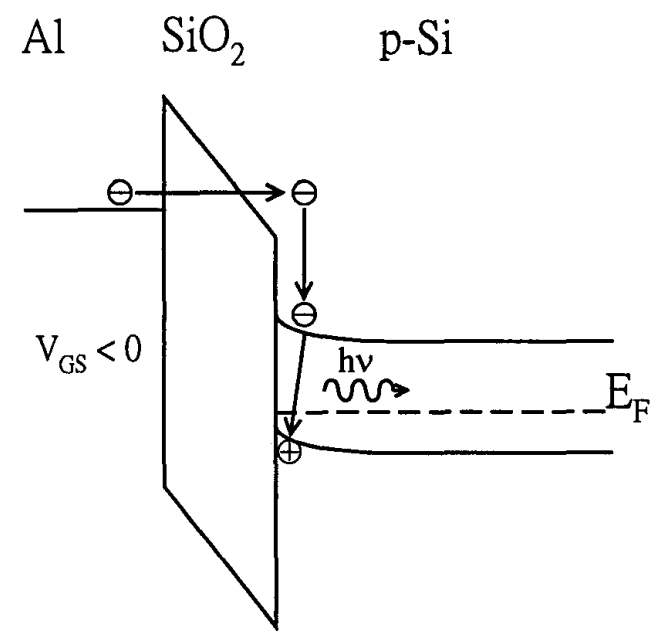

Fig. 3. The electron tunneling current and photon emission for NMOS at negative gate bias.

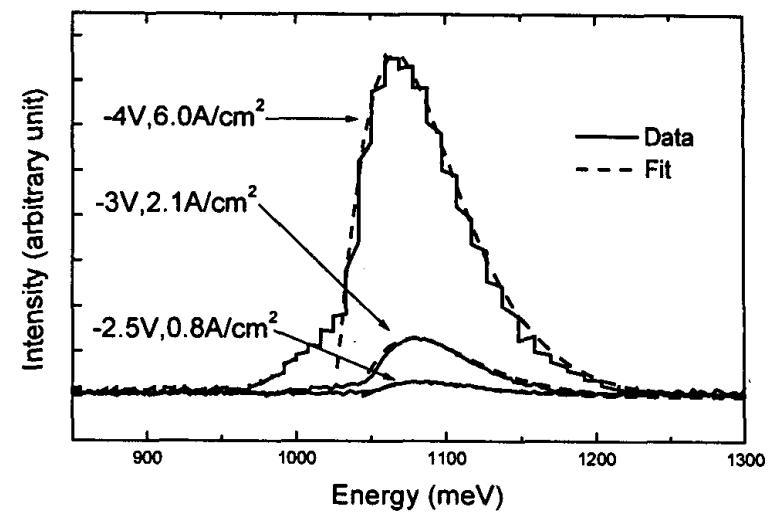

Fig. 4. Emission spectra of NMOS tunneling light emitting diodes as well as theoretical fitting.

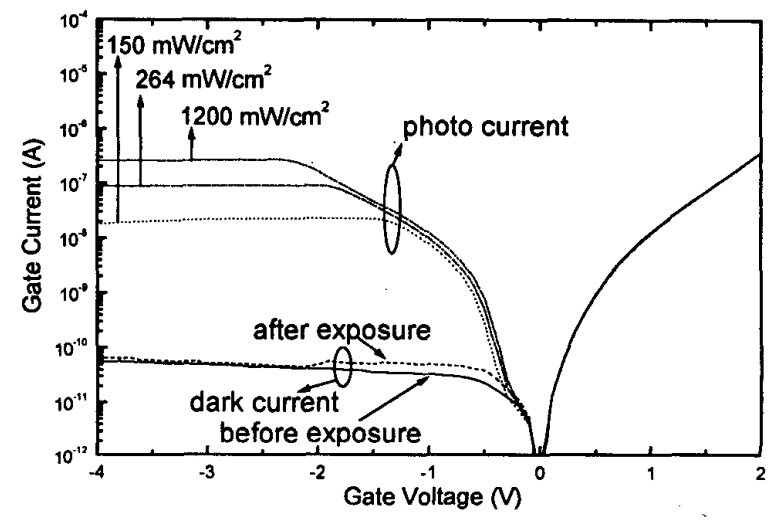

Fig. 5. Photo and dark currents of a PMOS tunneling diode at negative gate bias. Device size is $3 \times 10^{-4} \mathrm{~cm}^{2}$. Note the large saturation voltage of photo current $(\sim-2 \mathrm{~V})$ may be due to large valence band discontinuity $\left(\Delta \mathrm{E}_{\mathrm{V}}=4.5 \mathrm{eV}\right)$

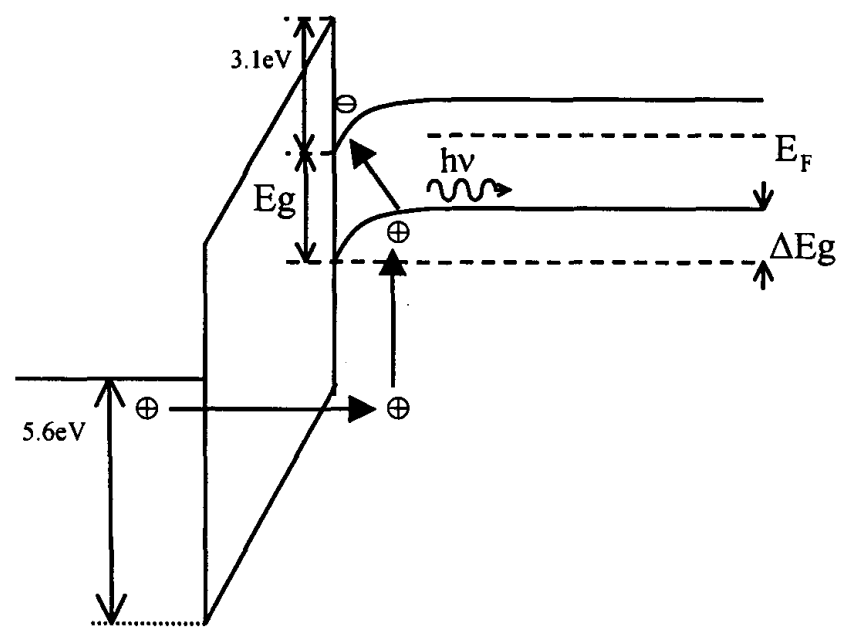

Fig. 6. A schematic band diagram to explain the band gap reduction.

31.3.3 


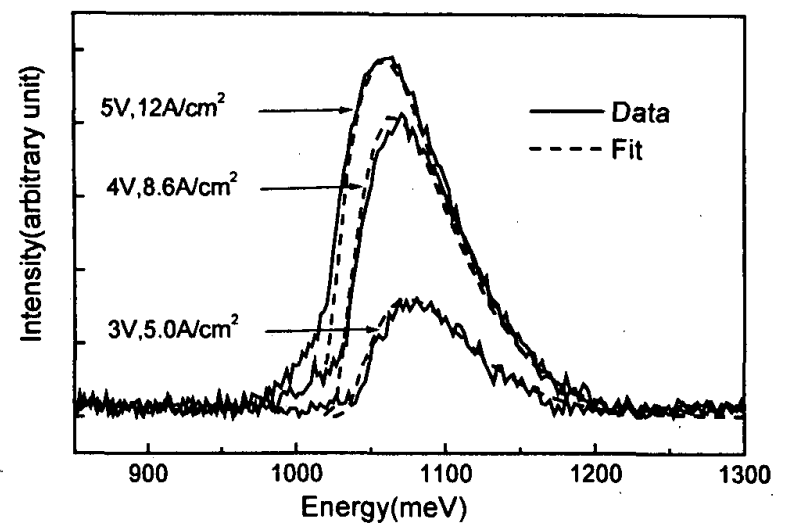

Fig. 7. Emission spectra of PMOS tunneling light emitting diodes as well as theoretical fitting.

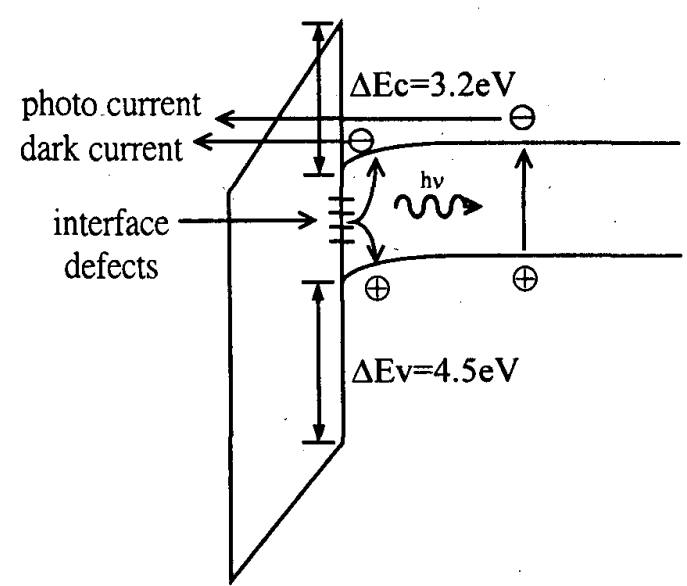

Fig. 8. The operation principle for a NMOS tunneling light detector at positive gate bias.

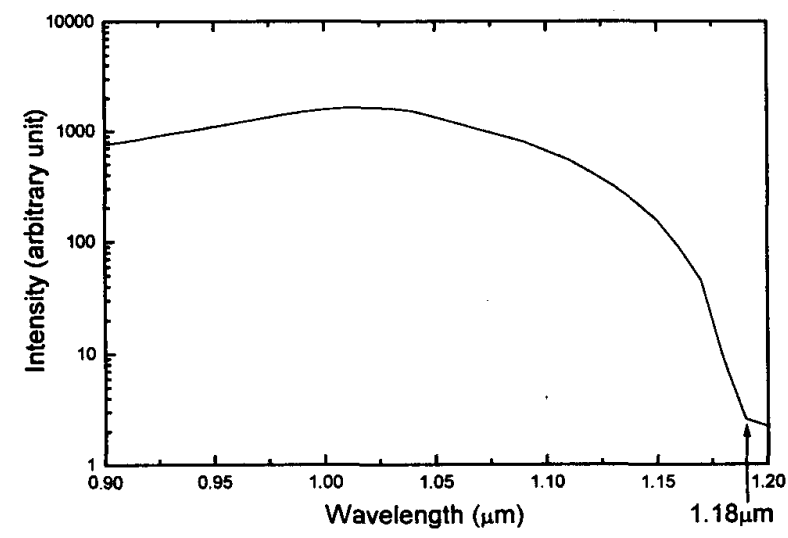

Fig. 9. The detection spectrum of a NMOS tunneling detector bias at zero bias. The tail at 1.1 to $1.18 \mu \mathrm{m}$ may be due to the interface states and surface band bending.

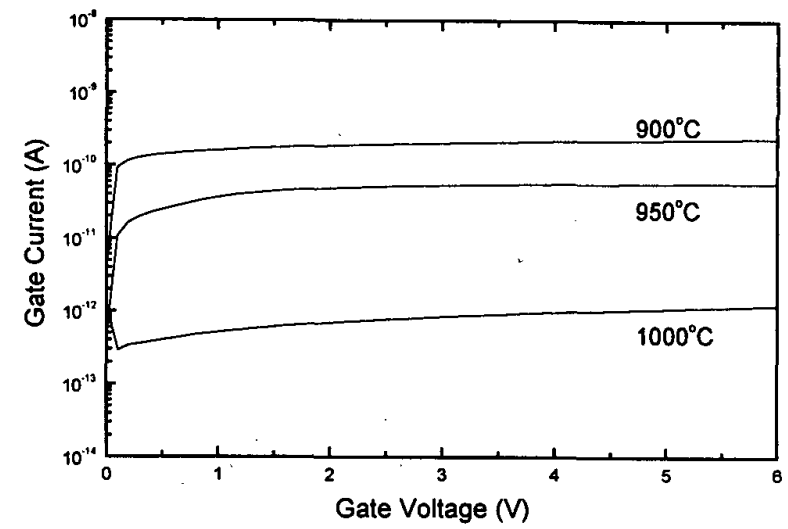

Fig. 10. The dark current of NMOS tunneling light detectors with oxide grown at different temperature. High growth temperature can decrease the dark current. The current spike around $0 \mathrm{~V}$ for $1000^{\circ} \mathrm{C}$ sample may be due to the tunneling through the interface states

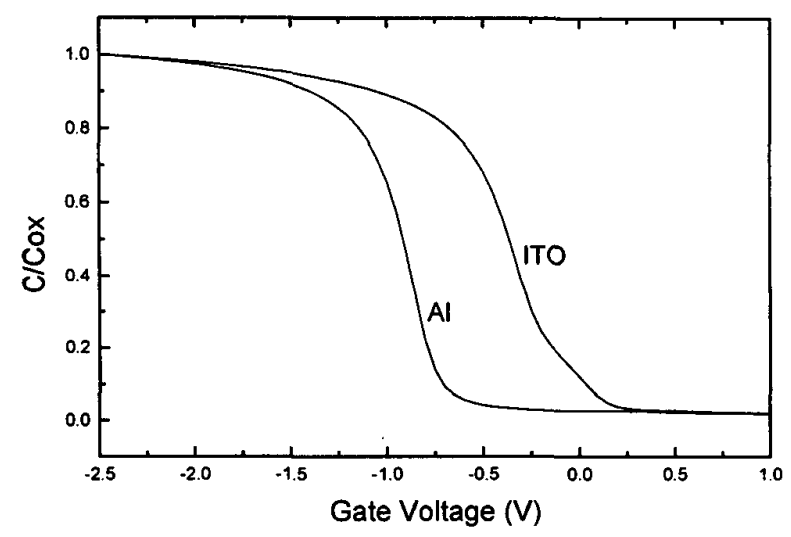

Fig. 11. C-V plots of ITO gate and Al gate NMOS devices. The work function of ITO is about $0.5 \mathrm{eV}$ lower than Al. This sample is not the same one in Fig. 2

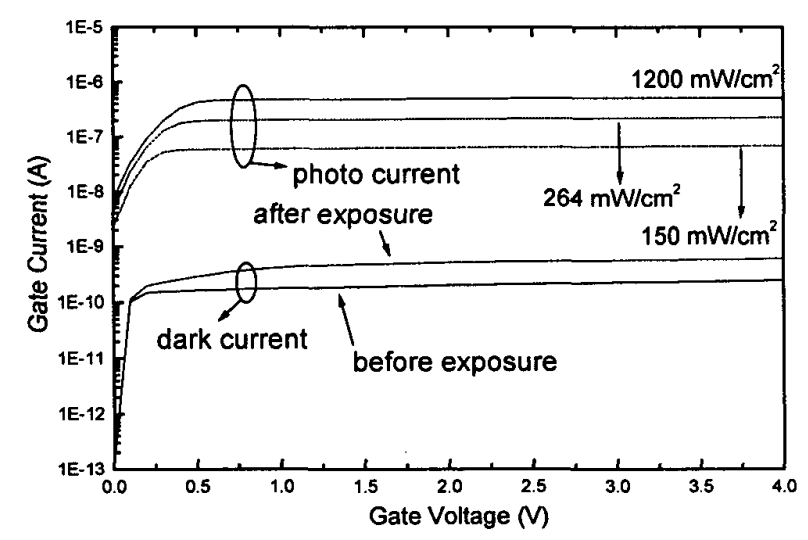

Fig. 12. Photo and dark currents of a NMOS tunneling diode using a ITO gate at positive bias. The photo current increases by a factor of 20 , compared to the $\mathrm{Al}$ gate.

\subsection{4}

